Pacific Journal of Mathematics

ON FINITE LEFT LOCALIZATIONS 


\title{
ON FINITE LEFT LOCALIZATIONS
}

\author{
R. S. CUNNINGHAM
}

Of the several types of noncommutative quotient rings, finite left localizations have structure most like that of the original ring. This paper examines finite left localizations from two points of view: As rings of quotients with respect to hereditary torsion classes, and as endomorphism rings of finitely generated projective modules. In the first case, finite left localizations are shown to be the rings of quotients with respect to perfect TTF-classes. In the second, they are shown to be the double centralizers of finite projectors.

The first characterization (Corollary 1.2) shows that finite left localizations may be realized as endomorphism rings of finitely generated projective idempotent ideals. It follows that every left localization of a right perfect ring is finite.

Finally, the finite projectors of the second characterization (Corollary 2.2) are shown to have endomorphism rings whose quotient ring structure is very closely related to that of the original ring. For example, every ring of left quotients of the endomorphism ring is Morita equivalent to a ring of quotients of the original ring.

1. Perfect TTF-classes. For an associative ring $R$ with identity let ${ }_{R} \mathscr{C l}$ denote the category of unital left $R$-modules. A (hereditary) torsion class $\mathscr{T} \leqq{ }_{R} \mathscr{C l}$ is a nonvoid class of left $R$-modules closed under taking submodules, factor modules, extensions, and arbitrary direct sums. Corresponding to a torsion class $\mathscr{T}$ is a torsion-free class $\mathscr{F}=\left\{M \in_{R} \mathscr{C l} \mid \operatorname{Hom}_{R}(N, M)=0 \forall N \in \mathscr{T}\right\}$ and a topologizing filter of left ideals $f=\{I$ left ideals of $R \mid R / I \in \mathscr{T}\}$. A torsion class determines a localization functor $L_{\mathscr{T}}$ from fro $_{R}$ to the quotient category $_{R} \mathscr{M} / \mathscr{T}($ see $[13, \S 2])$ given by $L_{\mathcal{J}}(M)=\underset{\vec{I} \in f}{\lim }=\operatorname{Hom}_{R}(I, M / t(M))$ where $t(M)$ is the $\mathscr{T}$-torsion submodule of $M$. This localization functor is covariant, additive, and left exact, and there is a natural transformation $\sigma_{M}: M \rightarrow L_{\mathcal{F}}(M)$ whose kernel is $t(M)$. The object $Q=L_{\sigma}(R)$ is a ring, called the ring of left quotients of $R$ with respect to $\mathscr{T}$, and the map $\sigma_{R}$ is a ring homomorphism. A torsion class $\mathscr{T}$, or its ring of quotients $Q$, is called faithful if $t(R)=0$. For more details, see [13] or [14].

Some special types of torsion classes will be of interest to us. Stenström [13, §13] calls a topologizing filter $f$ perfect in case $Q \cdot I=$ $Q$ for every left ideal $I \in f$ (where $Q$ is viewed as an $R$-module). We will call a torsion class $\mathscr{T}$ perfect if its associated filter is per- 
fect. These torsion classes have been characterized in [13, Theorem 13.1] and [14, Theorem 3.2]. We will refer to these characterizations as needed.

Perfect torsion classes are important because they give the left localizations of a ring. A left localization of $R$ is a ring $Q$ and a ring homomorphism $\sigma: R \rightarrow Q$ such that $\sigma$ is an epimorphism in the category of rings which gives $Q$ the structure of a flat right $R$-module [7], [13]. Stenström [13, §13] shows that $\mathscr{T}$ is perfect if and only if $\sigma_{R}: R \rightarrow Q$ is a left localization of $R$ and $\mathscr{T}=\left\{M \in_{R} \mathscr{M} \mid Q \otimes{ }_{R} M=0\right\}$. Silver [12] further calls a left localization finite if $Q$ is finitely generated and projective as a right $R$-module.

The other type of useful torsion class is the TTF-class. $\mathscr{T}$ is a TTF-class if there is a (not necessarily hereditary) torsion class $\mathscr{C}$ for which $\mathscr{T}$ is the corresponding torsion-free class. These were characterized by Jans [8, Theorem 2.1] as those torsion classes whose associated filter contains a unique minimal idempotent ideal $J$.

Notice that if $\mathscr{T}$ is a TTF-class, the localization functor is just $L_{\sigma}(M)=\operatorname{Hom}_{R}(J, M / t(M))$ where $J$ is the minimal element in the corresponding filter.

The following theorem characterizes finite left localizations in terms of these torsion classes.

THEOREM 1.1. Let $\mathscr{T}$ be a hereditary torsion class for a ring $R$ with corresponding ring of left quotients $Q$. The following statements are equivalent:

(1) $\mathscr{T}$ is a perfect TTF-class,

(2) $Q$ is a finite left localization of $R$ and $\mathscr{T}=\left\{M \in_{R} \mathscr{M} \mid\right.$ $\left.Q \otimes{ }_{R} M=0\right\}$,

(3) the localization functor $L_{\sigma}$ is exact and commutes with both direct sums and direct products,

(4) $\mathscr{T}$ is a TTF-class for which the minimal element $J$ of the corresponding filter satisfies:

(a) ${ }_{R} J$ is finitely generated, and

(b) ${ }_{R} J$ is projective relative to the class of epimorphisms $\mathscr{E}=$ $\{A \rightarrow B \rightarrow 0 \mid A, B \in \mathscr{F}\}$.

Proof. $\quad(1) \Rightarrow(4)$. Since $\mathscr{T}$ is perfect, $f$ has a cofinal set of finitely generated left ideals [14, Theorem 3.2(a)]. Further, since $\mathscr{T}$ is TTF, $f$ has a minimal element. Thus this minimal element ${ }_{R} J$ is finitely generated. Moreover, if $f: A \rightarrow B$ is any epimorphism, $L_{\mathscr{T}}(f): L_{\mathscr{T}}(A) \rightarrow L_{\mathscr{T}}(B)$ is onto. Since $L_{\mathscr{T}}(M) \cong \operatorname{Hom}_{R}(J, M)$ if $M \in \mathscr{F}$, ${ }_{R} J$ is projective relative to $\mathscr{E}$.

$(4) \Rightarrow(3)$. Condition (a) shows that $L_{\mathscr{J}}$ commutes with direct sums, while $L_{\mathscr{T}}$ commutes with direct products since $\mathscr{T}$ is a TTF- 
class. Finally, a straightforward check shows that condition (b) makes $L$, right exact and thus exact.

$(3) \Rightarrow(2)$. Since $L$ is exact and commutes with direct sums, $\mathscr{T}$ is a perfect torsion class [13, Theorem 13.1(c)]. Thus $Q$ is a left localization of $R$ and $\mathscr{T}=\left\{M \in_{R} \mathscr{C l} \mid Q \otimes_{R} M=0\right\}$. In particular, $Q_{R}$ is flat. Moreover, since $L$, is equivalent to $Q \otimes_{R}($ ) [13, Theorem 13.1(e)] and commutes with direct products, [4, Lemmas 1.1 and 1.2] show that $Q_{R}$ is finitely generated and, in fact, finitely presented. Hence by [3], $Q_{R}$ is projective and is a finite left localization of $R$.

$(2) \Rightarrow(1)$. Since $Q_{R}$ is finitely generated and projective and $Q \cong$ $\operatorname{End}_{R}\left(Q_{R}\right)$ by [9, Theorem 7.1], $\mathscr{T}$ is a TTF-class by [5, Proposition 1.4]. Finally, $\mathscr{T}$ is perfect by Stenström's characterization of left localizations.

REMARK. It would be tempting to replace condition (2) of the theorem by the condition that $Q$ be a finite left localization of $R$. However, Example 2.4 below will show that this is impossible.

COROLlary 1.2. There is a one-to-one correspondence between the finite left localizations of $R$ and the perfect TTF-classes, given by corresponding to $Q$ the class $\left\{M \in_{R} / \mathcal{Q} \mid Q \otimes_{R} M=0\right\}$.

Proof. This follows from the above theorem, [13, Theorem 13.1(f)], and [5, Proposition 1.4].

The following corollary shows that if $\mathscr{T}$ is also faithful, we may strengthen condition (4(b)) above.

COROLlary 1.3. Let $\mathscr{T}$ be a faithful hereditary torsion class in ${ }_{R}$ ll. Then $\mathscr{T}$ is a perfect TTF-class if and only if the associated filter $f$ has a minimal element $J$ which is finitely generated and projective as a left ideal of $R$. Moreover, in this case $Q \cong \operatorname{End}_{R}\left({ }_{R} J\right)$.

Proof. The condition on ${ }_{R} J$ is clearly sufficient. Conversely, since ${ }_{R} R$ and ${ }_{R} J$ are both in $\mathscr{F}$, the usual epimorphism of a free module onto ${ }_{R} J$ is in $\mathscr{E}$. Since ${ }_{R} J$ is projective relative to $\mathscr{E}$, this map must split. Hence ${ }_{R} J$ is projective. The last statement follows since ${ }_{R} J \in \mathscr{F}$ and $R / J \in \mathscr{T}$, whence $L,(R) \cong \operatorname{Hom}_{R}(J, R) \cong \operatorname{Hom}_{R}(J, J)$.

REMARK. The last statement of the corollary extends Silver's remark on finite left localizations of Noetherian rings [12, p. 58] to the general case. This was already clear, however, from Morita's result that $Q \cong \operatorname{End}_{R}\left(Q_{R}\right)$ for left localizations $Q$ [9, Theorem 7.1].

We end this section with three corollaries about right perfect rings. These results depend on the fact that for right perfect rings, 
every hereditary torsion class of left $R$-modules is a TTF-class. See [1, Corollary 1.6] and [6, Theorem A].

CoROLlaRy 1.4. If $R$ is a right perfect ring, every left localization of $R$ is a finite left localization.

Proof. Every perfect torsion class is a perfect TTF-class.

COROLlaRY 1.5. If $R$ is a right perfect ring, the faithful left localizations of $R$ are precisely those rings of left quotients of $R$ for which the minimal element in the corresponding filter is an idempotent, faithful, two-sided ideal $R f R$ which is finitely generated and projective as a left $R$-module. In this case $Q$ is Morita equivalent to $f R f$.

Proof. Since $R$ is right perfect, every idempotent ideal of $R$ is of the form $R f R$ for $f$ an idempotent in $R$. The first statement then follows directly from Corollaries 1.3 and 1.4. Now $R f R$ is the trace ideal of $f R$ in $R$ (see $\S 2$ ) and is finitely generated and projective as a left ideal of $R$. Thus it follows from [11, Corollary 4] and [2, Theorem 3.2] that $f R$ is a left progenerator over $f R f \cong \operatorname{End}_{R}(f R)$. Since $Q$ is isomorphic to the $f R f$-endomorphism ring of $f R[5$, Theorem 2.1], $Q$ is Morita equivalent to $f R f$.

COROLlaRY 1.6. The flat epimorphic hull of a right perfect ring is isomorphic to the ring of left $R$-endomorphisms of the minimal member of the set of ideals of $R$ described in the previous corollary.

Proof. The flat epimorphic hull is the maximal faithful left localization of $R$. The result then follows from Corollaries 1.5 and 1.3.

REMARK. This shows that the flat epimorphic hull of a right perfect ring can be concretely constructed. This contrasts with the general construction using a direct limit argument [7].

2. Finite projectors. In this section we examine the torsion class and ring of left quotients determined by a finitely generated projective module $P_{R}$ as in [5]. We ask when this torsion class is perfect. We will need some background and notation; details may be found in [5].

Let $P_{R}$ be a finitely generated projective right $R$-module with $S=\operatorname{End}_{R}\left(P_{R}\right)$. The trace ideal $T$ of $P_{R}$ is $T=\Sigma f(P)$ as $f$ ranges over $\operatorname{Hom}_{R}(P, R)$. The trace $T$ is an idempotent two-sided ideal of $R$ such that $P T=P$. The monule $P_{R}$ determines a hereditary tor- 
sion class $\mathscr{T}_{T}=\left\{M \in_{R} \mathscr{L C} \mid P \otimes_{R} M=0\right\}$; its lassociated filter is $f_{T}=$ $\{I$ left ideal of $R \mid I \supseteqq T\}$. Thus $\mathscr{T}_{T}$ is always a TTF-class. We denote the corresponding torsion-free class by $\mathscr{F}_{T}$, localization by $L_{T}$, and ring of left quotients by $Q_{T}$.

The bimodule ${ }_{S} P_{R}$ determines functors

$$
F={ }_{S} P_{R} \otimes{ }_{R}(\quad):{ }_{R} \mathscr{C l} \longrightarrow{ }_{S} \not l C
$$

and

$$
H=\operatorname{Hom}_{S}\left({ }_{s} P_{R},-\right):{ }_{s} \mathscr{M l} \longrightarrow{ }_{R} \sim / C
$$

such that $F$ is the left adjoint of $H$. Then the localization functor $L_{T}$ is naturally equivalent to the functor $H F$; thus $Q_{T}=L_{T}(R) \cong$ $H F(R)=\hat{R}$, the double centralizer of $P_{R}$, as rings. We will consistently denote this ring by $\hat{R}$.

THeORem 2.1. Let $R$ be a ring, $P_{R}$ be a finitely generated projective right $R$-module, and $S=\operatorname{End}_{R}\left(P_{R}\right)$. The following statements are equivalent:

(1) $\mathscr{T}_{T}$ is a perfect torsion class,

(2) ${ }_{s} P$ is finitely generated and projective,

(3) the functor $H$ is exact and commutes with direct sums.

Proof. $\quad(1) \Rightarrow(3)$. Let $\mathscr{T}_{T}$ be a perfect torsion class. Then the functor $L_{T}$, naturally equivalent to $H F$, is exact and commutes with direct sums. We claim this implies that $H$ is exact. Let $0 \rightarrow N^{\prime} \rightarrow$ $N \stackrel{\delta}{\longrightarrow} N^{\prime \prime} \rightarrow 0$ be exact in ${ }_{S}$ /l. Then $0 \rightarrow H\left(N^{\prime}\right) \rightarrow H(N) \stackrel{H(\delta)}{\longrightarrow} H\left(N^{\prime \prime}\right) \rightarrow$ coker $H(\delta) \rightarrow 0$ is exact in ${ }_{R} \not l$. Since $H F$ is exact, $0 \rightarrow H F H\left(N^{\prime}\right) \rightarrow$ $H F H(N) \rightarrow H F H\left(N^{\prime \prime}\right) \rightarrow H F($ coker $H(\delta)) \rightarrow 0$ is exact. Since $F H$ is naturally equivalent to the identity functor on s.ll, $F(\operatorname{coker} H(\delta))=$ 0 and $0 \rightarrow H\left(N^{\prime}\right) \rightarrow H(N) \stackrel{H(\delta)}{\longrightarrow} H\left(N^{\prime \prime}\right) \rightarrow 0$ is exact. Finally, since the trace ideal $T$ is the minimal element in the filter $f_{T}$, Theorem 1.1(4) shows that ${ }_{R} T$ is finitely generated. Then [11, Corollary 4] shows that ${ }_{S} P$ is finitely generated so that $H$ commutes with direct sums.

$(3) \Rightarrow(1)$. This is clear since $H F$ must be exact and commute with direct sums.

$(3) \Rightarrow(2)$. Since $H$ is exact, ${ }_{s} P$ must be projective. Moreover, since (3) is equivalent to (1), ${ }_{S} P$ is finitely generated.

$(2) \Rightarrow(3)$. This is clear.

Condition (2) of Theorem 2.1 is obviously equivalent to the condition that the functor $F={ }_{S} P_{R} \otimes_{R}(\quad)$ take finitely generated projective modules to finitely generated projective modules. Thus we follow Anderson [2] and call a finitely generated projective module satisfying the conditions of Theorem 2.1 a finite projector. We immediately get one more characterization of finite left localizations. 
Corollary 2.2. A ring of left quotients $Q$ of $R$ is a finite left localization of $R$ if and only if $Q$ is canonically isomorphic to the double centralizer $\hat{R}$ of a finite projector $P_{R}$.

Proof. Let $Q$ be a finite left localization of $R$. By [9, Theorem 7.1], $Q \cong \operatorname{End}_{R}\left(Q_{R}\right)$. Hence $Q_{R}$ is a finite projector and $Q$ is clearly its double centralizer. Conversely, if $P_{R}$ is a finite projector, $\mathscr{T}_{T}$ is a perfect TTF-class. Thus $\hat{R} \cong Q$ is a finite left localization of $R$ by Theorem 1.1.

The many characterizations of perfect torsion classes give rise to many for finite projectors. The following corollary lists a few; proofs are straightforward and easily seen from [5], [13], [14], or Theorem 1.1, and are thus omitted.

Corollary 2.3. For $R, P$, and $S$ as in Theorem 2.1, the following statements are equivalent.

(1) $P_{R}$ is a finite projector,

(2) ${ }_{R} T$ is finitely generated and is projective relative to the class of epimorphisms $\left\{A \rightarrow B \rightarrow 0 \mid A, B \in \mathscr{F}_{T}\right\}$,

(3) ${ }_{S} P$ is a progenerator,

(4) $\hat{R}$ and $S$ are Morita equivalent via ${ }_{S} P_{\hat{R}} \cong{ }_{S} P_{R} \otimes{ }_{R} \hat{R}$,

(5) $\hat{R} \cdot T=\hat{R}$,

(6) $L_{T}(M) \cong \hat{R} \otimes{ }_{R} M$ for all ${ }_{R} M$,

(7) every left $\hat{R}$-module is in $\mathscr{F}_{T}$ viewed as an $R$-module.

In view of Corollary 2.2, one might expect to add to this list the condition that $\hat{R}$ is a finite left localization of $R$. The following example shows that this condition is not sufficient.

EXAMPLE 2.4. Let $S$ be a ring with a nonprojective left $S$ module ${ }_{S} X$, let $P=S \otimes X$, and let $R=\operatorname{End}_{S}\left({ }_{S} P\right)$. Then $P_{R}$ is finitely generated and projective since ${ }_{S} P$ is a left $S$-generator. Further, $S \cong \operatorname{End}_{R}\left(P_{R}\right)$ since generators have the double centralizer property. Hence $R=\hat{R}$ clearly, and $\hat{R}$ is a finite left localization of $R$. But ${ }_{S} P$ is not projective; thus $P_{R}$ cannot be a finite projector.

The following two propositions give us some sources of finite projectors.

Proposition 2.5. If $R$ is left hereditary and left Noetherian, every finitely generated projective right $R$-module is a finite projector.

Proof. If $T$ is the trace ideal of $P_{R}$, then ${ }_{R} T$ is finitely generated and projective.

Recall that Anderson [2] calls $P_{R}$ a perfect projector (perfect 
injector) if the functor $F=P \otimes_{R}(\quad)$ preserves projective covers (injective hulls). See also [10].

Proposition 2.6. If $R$ is semiperfect and $P_{R}$ is a perfect projector, then $P_{R}$ is a finite projector.

Proof. By [10, Theorem 3.3] there is an idempotent $e \in R$ with $T=R e$. Since ${ }_{R} T$ is finitely generated and projective, $P_{R}$ is a finite projector.

The converse of this is false; there are triangular matrix ring examples which show that finite projectors need not be perfect projectors and that there is generally no relation between finite projectors and perfect injectors.

3. Correspondence of torsion classes and quotient rings. In this section we characterize finite projectors by special properties of certain correspondences from [5]. We begin with some background on these correspondences.

Let $P_{R}$ be a finitely generated projective right $R$-module with $S=\operatorname{End}_{R}\left(P_{R}\right)$. Let $F$ be the functor $F(M)={ }_{S} P \otimes{ }_{R} M:{ }_{R} \mathscr{C} \rightarrow{ }_{S} \mathscr{M}$ as in the last section. For a hereditary torsion class $\mathscr{T}_{R}$ in $R \mathscr{C l}$, define

$$
F\left(\mathscr{T}_{R}\right)=\left\{N \in \in_{S} \mathscr{C l} \mid N \cong F(M) \text { for some } M \in \mathscr{T}_{R}\right\}
$$

and for a hereditary torsion class $\mathscr{T}_{S}$ in ${ }_{s} \mathscr{C l}$, define

$$
F \leftarrow\left(\mathscr{T}_{S}\right)=\left\{M \in_{R} \mathscr{R C} \mid F(M) \in \mathscr{T}_{s}\right\} .
$$

The mappings $\mathscr{T}_{R} \rightarrow F\left(\mathscr{T}_{R}\right)$ and $\mathscr{T}_{S} \rightarrow F^{\leftarrow}\left(\mathscr{T}_{S}\right)$ are inclusion-preserving, inverse one-to-one correspondences between the hereditary torsion classes in ${ }_{S} \mathscr{C l}$ and those hereditary torsion classes in ${ }_{R} \mathscr{M l}$ which contain $\mathscr{T}_{T}=\left\{M \in_{R} \mathscr{C} \mid F(M)=0\right\}$. Further, if $Q_{R}$ and $Q_{S}$ are rings of left quotients of $R$ and $S$ with respect to hereditary torsion classes $\mathscr{T}_{R}$ and $\mathscr{T}_{S}$ which correspond, then $P \otimes_{R} Q_{R}$ is a finitely generated and projective right $Q_{R}$-module with $Q_{S} \cong \operatorname{End}_{Q_{R}}\left(P \otimes_{R} Q_{R}\right)$ and $Q_{R} \cong$ $\operatorname{End}_{Q_{S}}\left(P \otimes_{R} Q_{R}\right)$.

THEOREM 3.1. Let $R$ be a ring, $P_{R}$ be a finitely generated projective right $R$-module, and $S=\operatorname{End}_{R}\left(P_{R}\right)$. The following statements are equivalent:

(1) $P_{R}$ is a finite projector,

(2) the correspondence of torsion classes above yields a one-toone correspondence of the perfect torsion classes in ${ }_{s} \mathscr{M}$ with the perfect torsion classes in ${ }_{R} \mathscr{M l}$ containing $\mathscr{T}_{T}$, 
(3) if $Q_{R}$ and $Q_{S}$ are rings of left quotients with respect to torsion classes which correspond as above, then $Q_{R}$ and $Q_{S}$ are Morita equivalent via the module $P \otimes_{R} Q_{R} \cong Q_{S} \otimes_{S} P$.

Proof. $\quad(1) \Rightarrow(2)$. Let $\mathscr{T}_{R}$ and $\mathscr{T}_{S}$ correspond, let $\mathscr{T}_{R}$ be perfect, and let $N$ be a left $Q_{S}$-module. Since $Q_{S} \cong \operatorname{End}_{Q_{R}}\left(P \otimes_{R} Q_{R}\right)$, there is a left $Q_{R}$-module $M$ with $\left(P \otimes_{R} Q_{R}\right) \otimes_{Q_{R}} M \cong P \otimes_{R} M \cong N$. Since $\mathscr{T}_{R}$ is perfect, $M \in \mathscr{F}_{R}$, the corresponding torsion-free class; hence by [5, Proposition 3.4], $N \in \mathscr{F}_{s}$. Since a hereditary torsion class is perfect just in case every module over the ring of quotients is torsion-free [14, Theorem 3.2(d)], $\mathscr{T}_{S}$ is perfect.

Conversely, let $\mathscr{T}_{S}$ be perfect and let $M$ be a left $Q_{R}$-module. Since $\mathscr{T}_{R} \supseteqq \mathscr{T}_{T}$, the natural map $R \rightarrow Q_{R}$ can be extended to an $R$ homomorphism $\hat{R} \rightarrow Q_{R}$. An elementary argument shows that this map is, in fact, a ring homomorphism. Thus we may view $M$ as a left $\hat{R}$-module. Since $\mathscr{T}_{T}$ is perfect, $M \in \mathscr{F}_{T}$. Further, $P \otimes_{R} M \cong$ $\left(P \otimes_{R} Q_{R}\right) \otimes_{Q_{R}} M$ is a left $Q_{S}$-module, and is thus in $\mathscr{F}_{S}$. Again by [5, Proposition 3.4], $M \in \mathscr{F}_{R}$. Thus $\mathscr{T}_{R}$ is perfect.

$(2) \Longrightarrow(1)$. Since $\{0\} \subseteq s \mathscr{C l}$ is clearly perfect, and $\{0\}$ corresponds to $\mathscr{T}_{T}, \mathscr{T}_{T}$ is perfect. Hence $P_{R}$ is a finite projector.

$(1) \Rightarrow(3)$. If $P_{R}$ is a finite projector, ${ }_{S} P$ is a progenerator. Hence by [5, §4], $P \otimes_{R} Q_{R} \cong Q_{S} \otimes_{S} P$ is a left $Q_{S}$-progenerator and $Q_{R} \cong$ $\operatorname{End}_{Q_{S}}\left(Q_{S} \otimes_{S} P\right)$. Thus $Q_{R}$ and $Q_{S}$ are Morita equivalent.

$(3) \Rightarrow(1)$. This is clear by Corollary 2.3.

\section{REFERENCES}

1. J. S. Alin and E. P. Armendariz, TTF-classes over perfect rings, J. Aust. Math. Soc., 11 (1970), 499-503.

2. F. W. Anderson, Endomorphism rings of projective modules, Math. Z., 111 (1969), 322-332.

3. S. U. Chase, Direct products of modules, Trans. Amer. Math. Soc., 97 (1960), 457-473.

4. R. R. Colby and E. A. Rutter, Jr., ח-flat and II-projective modules, Arch. der Math., 22 (1971), 246-251.

5. R. S. Cunningham, E. A. Rutter, Jr., and D. R. Turnidge, Rings of quotients of endomorphism rings of projective modules, Pacific. J. Math., 41 (1972), 647-668.

6. V. Dlab, A characterization of perfect rings, Pacific. J. Math., 33 (1970), 79-88.

7. G. D. Findlay, Flat epimorphic extensions of rings, Math. Z., 118 (1970), 281-288.

8. J. P. Jans, Some aspects of torsion, Pacific. J. Math., 15 (1965), 1249-1259.

9. K. Morita, Localizations in categories of modules I, Math. Z., 114 (1970), 121-144. 10. E. A. Rutter, Jr., Perfect Projectors and Perfect Injectors. Ring Theory, Academic Press, New York-London, 1972.

11. F. L. Sandomierski, Modules over the endomorphism ring of a finitely generated projective module, Proc. Amer. Math. Soc., 31 (1972), 27-31.

12. L. Silver, Noncommutative localizations and applications, J. Algebra, 7 (1967), $44-76$. 
13. B. Stenström, Rings and Modules of Quotients, Springer, Lecture Notes in Mathematics, 237, 1971.

14. C. L. Walker and E. A. Walker, Quotient categories and rings of quotients, Rocky Mountain J. Math., 2 (1972), 513-555.

Received January 8, 1973.

THE UNIVERSITY OF KANSAS 



\section{PACIFIC JOURNAL OF MATHEMATICS}

\section{EDITORS}

RICHARD ARENS (Managing Editor)

University of California

Los Angeles, California 90024
J. DUGUNDJI*

Department of Mathematics

University of Southern California

Los Angeles, California 90007

D. Gilbarg and J. Milgram

Stanford University

Stanford, California 94305
University of Washington

Seattle, Washington 98105

ASSOCIATE EDITORS
E. F. BECKENBACH
B. H. NeumanN
F. WoLF
K. YosHIDA

\section{SUPPORTING INSTITUTIONS}

\author{
UNIVERSITY OF BRITISH COLUMBIA \\ CALIFORNIA INSTITUTE OF TECHNOLOGY \\ UNIVERSITY OF CALIFORNIA \\ MONTANA STATE UNIVERSITY \\ UNIVERSITY OF NEVADA \\ NEW MEXICO STATE UNIVERSITY \\ OREGON STATE UNIVERSITY \\ UNIVERSITY OF OREGON \\ OSAKA UNIVERSITY
}

\author{
UNIVERSITY OF SOUTHERN CALIFORNIA \\ STANFORD UNIVERSITY \\ UNIVERSITY OF TOKYO \\ UNIVERSITY OF UTAH \\ WASHINGTON STATE UNIVERSITY \\ UNIVERSITY OF WASHINGTON
* * * *
AMERICAN MATHEMATICAL SOCIETY \\ NAVAL WEAPONS CENTER
}

The Supporting Institutions listed above contribute to the cost of publication of this Journal, but they are not owners or publishers and have no responsibility for its content or policies.

Mathematical papers intended for publication in the Pacific Journal of Mathematics should be in typed form or offset-reproduced, (not dittoed), double spaced with large margins. Underline Greek letters in red, German in green, and script in blue. The first paragraph or two must be capable of being used separately as a synopsis of the entire paper. Items of the bibliography should not be cited there unless absolutely necessary, in which case they must be identified by author and Journal, rather than by item number. Manuscripts, in duplicate if possible, may be sent to any one of the four editors. Please classify according to the scheme of Math. Rev. Index to Vol. 39. All other communications to the editors should be addressed to the managing editor, or Elaine Barth, University of California, Los Angeles, California, 90024.

100 reprints are provided free for each article, only if page charges have been substantially paid. Additional copies may be obtained at cost in multiples of 50 .

The Pacific of Journal Mathematics is issued monthly as of January 1966. Regular subscription rate: $\$ 72.00$ a year (6 Vols., 12 issues). Special rate: $\$ 36.00$ a year to individual members of supporting institutions.

Subscriptions, orders for back numbers, and changes of address should be sent to Pacific Journal of Mathematics, 103 Highland Boulevard, Berkeley, California, 94708.

PUBLISHED BY PACIFIC JOURNAL OF MATHEMATICS, A NON-PROFIT CORPORATION

Printed at Kokusai Bunken Insatsusha (International Academic Printing Co., Ltd.), 270, 3-chome Totsuka-cho, Shinjuku-ku, Tokyo 160, Japan.

* C. R. DePrima California Institute of Technology, Pasadena, CA 91109, will replace J. Dugundji until August 1974.

Copyright (C) 1973 by Pacific Journal of Mathematics

Manufactured and first issued in Japan 


\section{Pacific Journal of Mathematics}

\section{Vol. 51, No. $2 \quad$ December, 1974}

Robert F. V. Anderson, Laplace transform methods in multivariate spectral theory .................................................. 339

William George Bade, Two properties of the Sorgenfrey plane . . . . . . . . . . . . 349

John Robert Baxter and Rafael Van Severen Chacon, Functionals on continuous

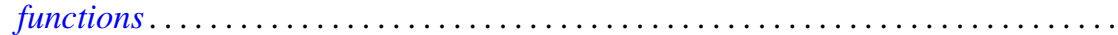

Phillip Wayne Bean, Helly and Radon-type theorems in interval convexity

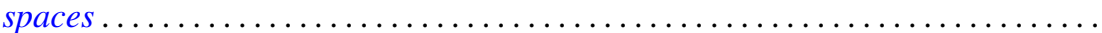

James Robert Boone, On k-quotient mappings $\ldots \ldots \ldots \ldots \ldots \ldots \ldots \ldots \ldots$

Ronald P. Brown, Extended prime spots and quadratic forms . . . . . . . . . . . .

William Hugh Cornish, Crawley's completion of a conditionally upper continuous lattice .............................................

Robert S. Cunningham, On finite left localizations ...................

Robert Jay Daverman, Approximating polyhedra in codimension one spheres

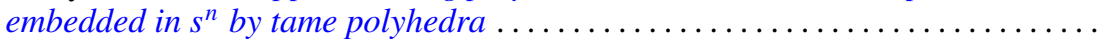

Burton I. Fein, Minimal splitting fields for group representations . . . . . . . . . . . .

Peter Fletcher and Robert Allen McCoy, Conditions under which a connected

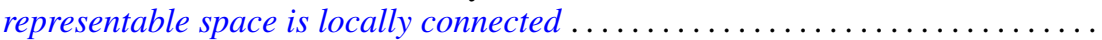

Jonathan Samuel Golan, Topologies on the torsion-theoretic spectrum of a noncommutative ring...

Manfred Gordon and Edward Martin Wilkinson, Determinants of Petrie matrices.

Alfred Peter Hallstrom, A counterexample to a conjecture on an integral condition for determining peak points (counterexample concerning peak points)........

E. R. Heal and Michael Windham, Finitely generated $F$-algebras with applications to Stein manifolds.

Denton Elwood Hewgill, On the eigenvalues of a second order elliptic operator in an unbounded domain ............................

Charles Royal Johnson, The Hadamard product of $A$ and $A^{*}$.

Darrell Conley Kent and Gary Douglas Richardson, Regular completions of Cauchy spaces.

Alan Greenwell Law and Ann L. McKerracher, Sharpened polynomial approximation

Bruce Stephen Lund, Subalgebras of finite codimension in the algebra of analytic functions on a Riemann surface. .

Robert Wilmer Miller, TTF classes and quasi-generators . .

Roberta Mura and Akbar H. Rhemtulla, Solvable groups in which every maximal partial order is isolated ....

Isaac Namioka, Separate continuity and joint continuity...

Alan Saleski, Entropy of self-homeomorphisms of statistical pseudo-metric

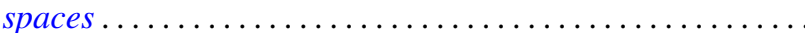

H. A. Seid, Cyclic multiplication operators on $L_{p}$-spaces .....

H. B. Skerry, On matrix maps of entire sequences ............

John Brendan Sullivan, A proof of the finite generation of invariants of a normal

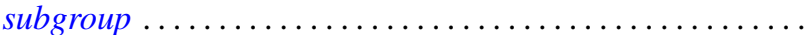

John Griggs Thompson, Nonsolvable finite groups all of whose local subgroups are

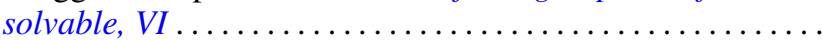

